This item was submitted to Loughborough's Research Repository by the author.

Items in Figshare are protected by copyright, with all rights reserved, unless otherwise indicated.

\title{
Bloch gain in dc-ac-driven semiconductor superlattices in the absence of electric domains
}

PLEASE CITE THE PUBLISHED VERSION

http://dx.doi.org/10.1103/PhysRevB.77.165330

PUBLISHER

(c) The American Physical Society

VERSION

VoR (Version of Record)

LICENCE

CC BY-NC-ND 4.0

\section{REPOSITORY RECORD}

Hyart, Timo, Kirill N. Alekseev, and Erkki V. Thuneberg. 2019. "Bloch Gain in Dc-ac-driven Semiconductor Superlattices in the Absence of Electric Domains". figshare. https://hdl.handle.net/2134/12813. 


\title{
Bloch gain in dc-ac-driven semiconductor superlattices in the absence of electric domains
}

\author{
Timo Hyart, ${ }^{1}$ Kirill N. Alekseev, ${ }^{1,2}$ and Erkki V. Thuneberg ${ }^{1}$ \\ ${ }^{1}$ Department of Physical Sciences, University of Oulu, P.O. Box 3000, FI-90014 Oulu, Finland \\ ${ }^{2}$ Department of Physics, Loughborough University, Looghborough LE11 3TU, United Kingdom \\ (Received 19 December 2007; revised manuscript received 15 March 2008; published 22 April 2008)
}

\begin{abstract}
We theoretically study the feasibility of amplification and generation of terahertz radiation in dc-ac-driven semiconductor superlattices in the absence of electric domains. We find that if in addition to a dc bias a strong terahertz pump field is applied, a Bloch gain profile for a small terahertz signal can be achieved under the conditions of a positive static differential conductivity. Here, the positive differential conductivity arises, similarly to the case of a large-signal amplification scheme [H. Kroemer, arXiv:cond-mat/0009311 (unpublished)], due to modifications in dc current density caused by the application of a high-frequency ac field [K. Unterrainer et al., Phys. Rev. Lett. 76, 2973 (1996)]. Whereas the sign of absorption at low and zero frequencies is sensitive to the ac fields, the gain profile in the vicinity of the gain maximum is robust. We suggest to use this ac-induced effect in a starter for a terahertz Bloch oscillator. Our analysis demonstrates that the application of a short terahertz pulse to a superlattice allows the suppression of the undesirable formation of electric domains and the achievement of a sustained large-amplitude operation of the dc-biased Bloch oscillator.
\end{abstract}

DOI: 10.1103/PhysRevB.77.165330

PACS number(s): 03.65.Sq, 73.21.Cd, 07.57.Hm, 72.30.+q

\section{INTRODUCTION}

Terahertz radiation $(0.3-10 \mathrm{THz})$ has many promising applications in different areas of science and technology such as space astronomy, wideband communications, and biosecurity. ${ }^{1}$ One of the main challenges in the development of terahertz technology is the construction of coherent, monochromatic, and miniature sources of terahertz radiation that can operate at room temperature. A great recent achievement was the development of quantum cascade lasers that operate at terahertz frequencies by employing quantum transitions between energy levels in multiple quantum well heterostructures. ${ }^{2}$ Continuous improvements in the design of terahertz quantum cascade lasers have allowed their operational temperature to be increased above $100 \mathrm{~K}^{3}$ However, it is certainly very difficult to maintain population inversion in a terahertz quantum cascade laser at room temperature.

Semiconductor superlattices (SLs), ${ }^{4}$ which are working in the miniband transport regime, attract much attention as an artificial nonlinear medium demonstrating a resonant interaction with terahertz radiation (for review, see Refs. 5-7). In the presence of dc bias, electrons from a single miniband of SL can perform transient terahertz Bloch oscillations, ${ }^{8,9}$ which decay at a picosecond time scale due to unavoidable scattering. ${ }^{10}$ In the stationary transport regime, when the scattering of the miniband electrons is an important factor, the possibility of inversionless amplification and generation of terahertz radiation in SLs has been recognized well after some seminal suggestions. ${ }^{4,11}$ Bloch oscillations can potentially lead to the amplification of a weak ac field at frequencies smaller than the Bloch frequency, whereas absorption occurs for frequencies that are larger than the Bloch frequency. ${ }^{11}$ If electrons can perform relatively many cycles of Bloch oscillations between scattering events, the maximum of the small-signal gain is achieved in the vicinity of the Bloch frequency. This Bloch gain profile, which is shaped as a familiar dispersion curve, has recently attracted a lot of attention because estimates predicted a significant terahertz gain near the Bloch frequency at room temperature. Moreover, the Bloch gain for terahertz signals with a large amplitude has also been estimated. ${ }^{12,13}$

Within the semiclassical picture, the physical mechanism of the high-frequency gain can be understood in terms of ballistic trajectories of electrons in the quasimomentum space. It was found that the miniband electrons are gathered into certain favorable trajectories forming electron bunches, which are eventually responsible for the negative absorption. ${ }^{14}$ Alternatively, the Bloch gain can be explained within theories that consider the scattering-assisted transitions between the Wannier-Stark states. ${ }^{15}$ It is likely that the second order scattering-assisted mechanism of gain is not restricted to the case of a single-band transport in SLs. The dispersive profile of optical gain has been recently observed in quantum cascade lasers. ${ }^{16}$

However, at least in the case of miniband transport in dc-biased SL, the small-signal gain profile extends to arbitrarily low frequencies, ${ }^{11}$ which in essence means that the dc differential conductivity is also negative. SLs with a static negative differential conductivity (NDC), similarly to the bulk semiconductors demonstrating the Gunn effect, ${ }^{17}$ are unstable against the development of space-charge instability. ${ }^{11,18}$ This electric instability eventually results in the formation of moving domains with a high electric field inside the SL. ${ }^{19}$ So far, the NDC-related instability has prevented an unambiguous observation of the net Bloch gain in long SLs.

Importantly, it is still potentially possible to avoid the formation of the electric domains in SL, if the amplified terahertz signal has enough large amplitude. In this case, as has been demonstrated for a particular set of amplitude and frequency, ${ }^{20}$ the dependence of the time-averaged current on the applied bias can have a positive slope for a proper choice of the operation point. This effect in SLs is similar to the so-called limited space-charge accumulation (LSA) mode of operation known in semiconductor devices with hot 
electrons. ${ }^{21-23}$ The finding ${ }^{20}$ can potentially be used for the construction of a terahertz amplifier with a large offset. However, to realize the terahertz oscillator, it is still necessary to understand how to avoid the destructive electric domains at small amplitudes in order to reach the large-amplitude regime supporting the stable operation of the device. This is known as the device-turn-on problem for a terahertz Bloch oscillator. $^{20}$

In recent years, a number of interesting suggestions for the realization of a small-signal Bloch gain in a SL were put forward. The first direction in this research area consists in the experimental investigations of the Bloch gain at short space or time scales, when the electric domains have not enough time to build up. An advanced nanostructure design, which is an array of short SLs, has been introduced to measure a frequency dependent crossover from loss to gain. ${ }^{24}$ Modern ultrafast optical techniques were applied to measure the Bloch gain in undoped superlattices during a short time window after a femtosecond optical excitation of carriers in the SL. ${ }^{25,26}$ Another interesting suggestion is to work with two-dimensional (2D) structures, where the electric domains are effectively more suppressed in comparison with the case of three-dimensional (3D) structures. ${ }^{27,28}$ With this aim, a lateral surface SL shunted by another SL (Ref. 29) has been introduced as an active medium for a future realization of the Bloch oscillator. ${ }^{30}$ At least theoretically, it is also possible to obtain a high-frequency gain in conditions of a positive dc differential conductivity (PDC) by engineering the miniband dispersion relations in SLs (Ref. 31) or by using a hot electron injection into a miniband. ${ }^{32}$

Our approach to the problem is inspired by the investigation of the ac gain feasibility in the weakly coupled and low-doped SLs, where the electric transport is determined by a sequential tunneling between the wells. ${ }^{33}$ At relatively low temperatures an additional peak appears in the voltagecurrent characteristic due to the presence of impurity bands. The basic idea ${ }^{33}$ is to put a working point at the part of this peak with a positive slope. Then, following the Tucker formula for absorption ${ }^{34}$ and its geometric interpretation, the existence of a high-frequency gain is not indispensably connected to the operation at static NDC. One of our aims here is to show how a similar approach works in the case of strongly coupled SLs, where additional peaks can arise due to an action of an auxiliary ac field.

In this paper, we theoretically show how to reach gain for terahertz signals with both small and large amplitudes in a single miniband SL combining the action of constant and alternating electric fields. We focus on the requirements for gain under the conditions of the electric stability. With this aim, we first suppose that the electric field, acting on the miniband electrons, is $E_{d c}+E_{\delta} \cos \omega t$ and re-examine the scheme of electrically stable large-amplitude $\left(E_{\delta}\right)$ operation of the Bloch oscillator. ${ }^{20}$ We demonstrate that the gain in conditions of static PDC exists in rather wide ranges of $\omega$ and $E_{\delta}$. In a real device, the ac probe field $E_{\delta} \cos \omega t$ should be the mode of a high- $Q$ resonator.

As the next step, we consider the action of the bichromatic field $E_{d c}+E_{a c} \cos \Omega t+E_{\delta} \cos \omega t$, where $E_{a c} \cos \Omega t$ is an additional external terahertz field (pump) and the amplitude of the probe field (resonator mode), $E_{\delta}$, is now small.
The pump $\Omega$ and probe $\omega$ frequencies are assumed to be incommensurate. The basic idea behind this part of our paper is to use the external ac field to suppress the electric instability in SL for some range of bias $E_{d c}$. We show that in the case of dc-ac-driven SL, the dispersive gain profile stays almost unaltered at high frequencies $\omega \simeq n \Omega(n=1,2, \ldots)$, although at small frequencies, the absorption can change its sign and become positive (see Fig. 6). Thus, terahertz gain without the electric instability can be achieved, and this bichromatic scheme can be potentially used to realize a terahertz generator. The expense we must pay for this new attractive possibility is the necessity to use the pump terahertz field.

We find that the physical mechanism of suppression of the electric instability in both these schemes is based on local modifications of the voltage-current $(V I)$ characteristics of the SL induced by the terahertz field. ${ }^{35,36}$ However, while in the large-amplitude mode of the Bloch oscillator such changes in the dc current are self-induced by the strong resonator field $E_{\delta} \cos \omega t$, in the bichromatic scheme, at least in the limit of a weak probe field $\left(E_{\delta} \rightarrow 0\right)$, they are mainly caused by the action of the external ac field.

Our analysis also shows that it is potentially possible to combine these two schemes of generation in a single device. Namely, we suggest to use the external field as a starter for the terahertz Bloch oscillator: The temporal action of the ac pump can provide both the necessary suppression of electric domains and the gain for the small-amplitude oscillations of the resonator mode before the field strength in this mode, $E_{\delta}$, can reach up to the amplitude supporting an electrically stable operation without an external pump. Since the incommensurability of frequencies is supposed, the pump field of the starter should not necessarily be monochromatic. Intensive, broadband terahertz pulses ( $\mathrm{T}$ rays) ${ }^{37}$ can be also used to ignite the stable large-amplitude operation of the superlattice Bloch oscillator. Thus, our theoretical research contributes to the solution of the device-turn-on problem of the canonic Bloch oscillator.

\section{NONLINEAR ELECTRON TRANSPORT AND THE CANONIC BLOCH OSCILLATOR}

Everywhere in this paper, we work within the semiclassical approach based on the use of the Boltzmann transport equation for the miniband with the tight-binding dispersion relation. ${ }^{5,6}$ We mainly use the standard approximation of a single constant relaxation time $\tau$ and employ an exact formal solution of the Boltzmann equation; ${ }^{38}$ the effects of two distinct relaxation times are discussed in the Appendix. We are interested in the electron dynamics under the action of a time-dependent field, which consists of constant and alternating parts, $E(t)=E_{d c}+E_{a l t}(t)$. In a general case, the ac part $E_{a l t}(t)$ can contain many incommensurate frequency components. We define the absorption of the probe ac field $E_{\delta} \cos \omega t$ in the SL miniband as

$$
\mathcal{A}(\omega)=\langle j(t) \cos \omega t\rangle_{t},
$$

where $j(t)$ is the current density induced in the SL by the total field $E(t)$. In a general case, the averaging $\langle\cdots\rangle_{t}$ is per- 
formed over an infinite time. The gain corresponds to $\mathcal{A}$ $<0$. In this section, we will consider only the monochromatic ac field:

$$
E(t)=E_{d c}+E_{\delta} \cos \omega t .
$$

For this field, averaging in Eq. (1) should be performed only over the period $T=2 \pi / \omega$.

The absorption (gain) $\alpha$ (in $\mathrm{cm}^{-1}$ ) is related to $\mathcal{A}$ as ${ }^{15}$

$$
\alpha=\frac{2}{n_{r} \epsilon_{0} c} \frac{\mathcal{A}}{E_{\delta}},
$$

where $\epsilon_{0}$ and $c$ are the permittivity and the speed of light in vacuum, and $n_{r}$ is the refractive index of the SL material. Whenever the gain overcomes the loss in the resonator, we have an oscillator. For the oscillator, the generated power density $\mathcal{P}$ inside the sample is

$$
\mathcal{P}=\mathcal{A} E_{\delta} .
$$

To estimate the gain and generated power in physical units, we will use everywhere in this paper the following parameters of a typical GaAs/AlAs SL: period $d=6 \mathrm{~nm}$, miniband width $\Delta=60 \mathrm{meV}$, density of electrons $N=10^{16} \mathrm{~cm}^{-3}$, and refractive index $n_{r}=\sqrt{13}$ (GaAs). We consider operation at room temperature. The characteristic scattering time of miniband electrons is $\tau \simeq 200 \mathrm{fs}$.

Before proceeding with the analysis of gain in the acdriven case, it is worth reminding the main nonlinear transport properties of a dc-biased SL. The dependence of the dc current density on the dc bias is given by the Esaki-Tsu formula ${ }^{4}$

$$
j_{d c}\left(e E_{d c} d\right)=j_{\text {peak }} \frac{2 e E_{d c} d / \Gamma}{1+\left(e E_{d c} d / \Gamma\right)^{2}} .
$$

Here, $\Gamma=\hbar / \tau$ and the peak current, corresponding to $E_{d c}$ $=E_{c r}\left[E_{c r} \equiv \hbar /(e d \tau)\right]$, is

$$
j_{\text {peak }}=\frac{e N v_{0}}{2} \frac{I_{1}\left(\frac{\Delta}{2 k_{b} T}\right)}{I_{0}\left(\frac{\Delta}{2 k_{b} T}\right)},
$$

where $v_{0}=\Delta d /(2 \hbar)$ is the maximal electron velocity in the miniband and $I_{k}(x)(k=0,1)$ are the modified Bessel functions. This temperature dependence of the dc current is in good agreement with the experiment. ${ }^{39}$ The dependence of $j_{d c}$ on $E_{d c}$ is shown in Fig. 1. Instead of the field strength variable $E_{d c}$, the Esaki-Tsu characteristic can be represented in terms of the Bloch frequency $\omega_{B}=e d E_{d c} / \hbar$ by using the equality $E_{d c} / E_{c r}=\omega_{B} \tau$. For $\omega_{B} \tau \sim 1$, the Bloch frequency belongs to the terahertz frequency range. ${ }^{4}$

The dc differential conductivity, $\sigma_{d c}=\partial j_{d c} / \partial E_{d c}$, defines the slope of the VI characteristics at the working point. For the Esaki-Tsu dependence[Eq. (3)], it is

$$
\sigma_{d c}\left(e E_{d c} d\right)=\frac{2 j_{\text {peak }}}{E_{c r}} \frac{1-\left(e E_{d c} d\right)^{2} / \Gamma^{2}}{\left[1+\left(e E_{d c} d\right)^{2} / \Gamma^{2}\right]^{2}} .
$$

If $E_{d c} / E_{c r}=\omega_{B} \tau>1$, then $\sigma_{d c}$ is negative, and therefore, the Esaki-Tsu characteristic demonstrates NDC.

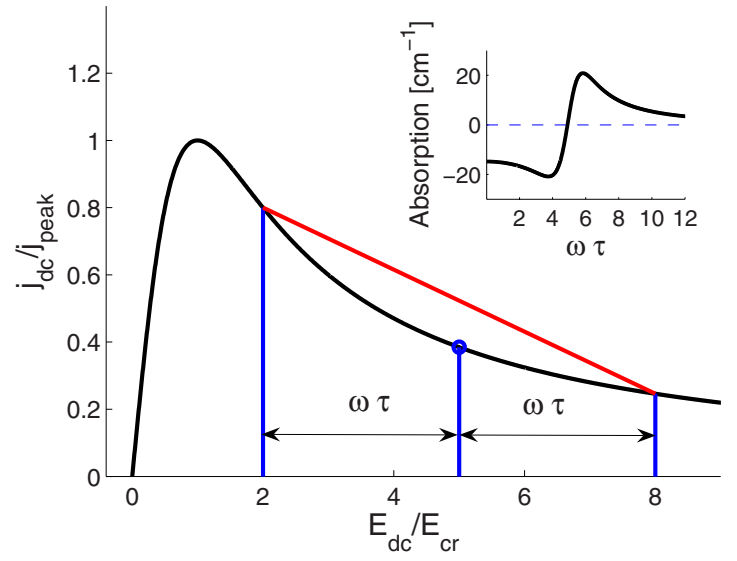

FIG. 1. (Color online) The Esaki-Tsu characteristic. The figure demonstrates the quantum derivative for the frequency $\omega=3 \tau^{-1}$ taken at the operation point $E_{d c}=5 E_{c r}$. Absorption at frequency $\omega$ is proportional to the difference quotient, Eq. (5), which is the slope of the inclined (red online) segment. Inset: Small-signal gain as a function of $\omega$ for $E_{d c}=5 E_{c r}$. The Bloch gain exists almost up to $\omega$ $\approx \omega_{B}=5 \tau^{-1}$ with the maximum of gain at $\omega \approx \omega_{B}-\tau^{-1}=4 \tau^{-1}$.

\section{A. Small-signal gain and electric instability in dc-biased superlattices}

We start with the consideration of the small-signal gain in a dc-biased superlattice [Eq. (2)]. In this case, the solution of the Boltzmann equation by a perturbation theory gives the well-known formula of Ktitorov et al. for absorption $\mathcal{A}(\omega) .{ }^{11}$ However, having in mind our future treatment of SL in biand polychromatic fields, it is instructive to introduce here the notion of a quantum derivative and briefly analyze the high-frequency gain by using this tool. The absorption [Eq. (1)] can be represented as the quantum derivative of the Esaki-Tsu current-field dependence [Eq. (3)] as ${ }^{6,33,40}$

$$
\mathcal{A}(\omega)=\frac{1}{2} \frac{j_{d c}\left(e E_{d c} d+\hbar \omega\right)-j_{d c}\left(e E_{d c} d-\hbar \omega\right)}{2 \hbar \omega} e E_{\delta} d .
$$

Figure 1 demonstrates the meaning of the quantum derivative (finite difference) in the case of a high-frequency probe field with $\hbar \omega=3 \Gamma(\omega \tau=3)$ and the Wannier-Stark spacing $\hbar \omega_{B}=5 \Gamma\left(E_{d c}=5 E_{c r}\right)$. Geometrically, the quantum derivative represents the slope of the segment with the length defined by the probe frequency $\omega$. The ends of the segment belong to the Esaki-Tsu curve and their locations are determined by the choice of the working point. In the quasistatic limit $\omega \tau$ $\ll 1$, we immediately get from Eq. (5) the well-known result that the absorption is proportional to the de differential conductivity at the operation point [Eq. (4)]. On the other hand, as is obvious from the figure, in order to provide a smallsignal gain in the canonic Bloch oscillator, the working point must be in the NDC portion of the Esaki-Tsu characteristic.

The frequency dependence of absorption, which was calculated using Eqs. (5) and (3), is shown in the inset of Fig. 1. It illustrates the dispersive profile of the Bloch gain at high frequencies $\omega \simeq \omega_{B}$ and also the existence of a negative absorption for $\omega \tau \rightarrow 0$. The frequency corresponding to the gain resonance can be most easily estimated for the choice of a working point satisfying $E_{d c} \gg E_{c r}$. As is obvious from the 
geometric meaning of Eq. (5), the maximum of gain is achieved when the left end of the inclined (red online) segment is located at the peak of the VI characteristic. Thus, the maximum of gain corresponds to the photon energy $\hbar \omega$ $\approx \hbar \omega_{B}-\Gamma\left(\omega \tau \approx \omega_{B} \tau-1\right)$. This gain resonance indicates a dissipative quantum nature of the Bloch gain profile: Asymmetry in the elementary acts of emission and absorption is caused by the scattering. However, one should always remember that the feasibility of high-frequency generation in the canonic Bloch oscillator is complicated by the NDCrelated electric instability.

\section{B. Photon-assisted peaks and large-signal gain}

In the case of that the probe field has a large amplitude, Eq. (2), the high-frequency gain is not necessarily connected to the presence of static NDC anymore because the ac field can open up new transport channels leading to the formation of photon-assisted peaks in the VI characteristic. ${ }^{35,36}$ Here, the dc current can be calculated with the help of photon replicas of the Esaki-Tsu current ${ }^{6}$

$$
j_{d c}^{\omega}\left(e E_{d c} d\right)=\sum_{l} J_{l}^{2}(\beta) j_{d c}\left(e E_{d c} d+l \hbar \omega\right)
$$

and the formula for absorption takes the form

$$
\mathcal{A}=\frac{1}{2} \sum_{l} J_{l}(\beta)\left[J_{l+1}(\beta)+J_{l-1}(\beta)\right] j_{d c}\left(e E_{d c} d+l \hbar \omega\right),
$$

where $J_{n}(x)$ are the Bessel functions, the summation is in infinite limits, $\beta=e E_{\delta} d / \hbar \omega$, and $j_{d c}$ is given by Eq. (3). We used the notation $j_{d c}^{\omega}$ to distinguish the dc current modified by the action of the ac field from the unmodified Esaki-Tsu current $j_{d c}$. By using Eq. (6), it is easy to calculate the dc differential conductivity, $\sigma_{d c}^{\omega}=\partial j_{d c}^{\omega} / \partial E_{d c}$,

$$
\sigma_{d c}^{\omega}=\sum_{l} J_{l}^{2}(\beta) \sigma_{d c}\left(e E_{d c} d+l \hbar \omega\right),
$$

where $\sigma_{d c}$ is given by formula (4).

Figure 2 shows the first photon-assisted peak in the dependence of $j_{d c}^{\omega}$ on $E_{d c}$ arising under the action of a strong ac field. Importantly, the left wing of the peak $\left(4<E_{d c} / E_{c r}\right.$ $<6)$ is characterized by PDC. PDC exists if the WannierStark spacing approximately equals the photon energy, $\left|\hbar \omega_{B}-\hbar \omega\right|<\Gamma$ (in other words, if $\left|E_{d c} / E_{c r}-\omega \tau\right|<1$ ).

Now, we want to demonstrate the feasibility of a largesignal gain in conditions of static PDC. With this aim, we choose the working point at the left wing of the first photonassisted peak in Fig. 2 at $\hbar \omega_{B}=5.5 \Gamma\left(E_{d c}=5.5 E_{c r}\right)$ and vary the amplitude of the ac field $E_{\delta}$. The absorption and, in our notations, power density will be negative if the SL can generate a high-frequency radiation. The dependence of the power density on $E_{\delta}$ is depicted in Fig. 3. In the same figure, we also indicated the range of $E_{\delta}$ for which NDC is realized at the working point. We see that the generation can be definitely complicated by the existence of electric instability at small probe amplitudes: A threshold value always exists, which the probe field amplitude must reach before the SL can switch to PDC. However, if the small-signal space-

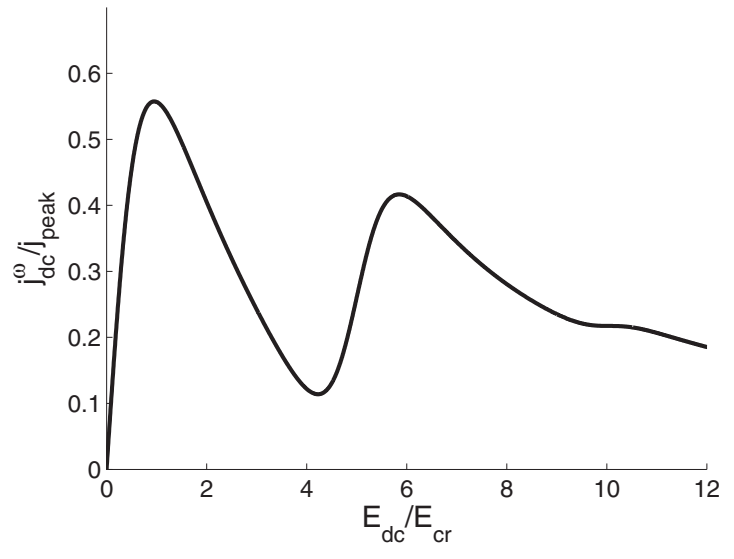

FIG. 2. VI characteristic of a superlattice modified by the strong monochromatic field with $E_{\delta}=5 E_{c r}$ and $\omega \tau=5$. Note the appearance of the photon-assisted peak centered at $E_{d c} / E_{c r}=6$. At this peak, the differential conductivity is positive for $4=\omega \tau-1<E_{d c} / E_{c r}<\omega \tau$ $+1=6$.

charge instability can be suppressed by some means, for example, by a very special SL design ${ }^{24}$ the generated power density at frequency $\omega / 2 \pi=5 \tau^{-1} / 2 \pi \approx 4 \mathrm{THz}$ can reach $\sim 40 \mathrm{MW} / \mathrm{cm}^{3}$ (Fig. 3). For a typical semiconductor SL, it corresponds to the power of $\sim 100 \mu \mathrm{W}$.

The whole ranges of bias $E_{d c}$ and probe amplitudes $E_{\delta}$, supporting simultaneously PDC and gain at the first photonassisted peak, are presented in Fig. 4 for one particular value of the photon energy, $\hbar \omega=5 \Gamma(\omega \tau=5)$. This figure shows that gain without NDC exists if $E_{\delta} \geqslant 2 E_{c r}$ and $\hbar \omega<\hbar \omega_{B}$ $<\hbar \omega+\Gamma\left(5<E_{d c} / E_{c r}<6\right)$.

Similarly, we also calculated the slope of the $j_{d c}^{\omega}\left(E_{d c}\right)$ dependence and the sign of absorption for many other probe frequencies. We summarize the requirements to obtain gain without NDC at the first photon-assisted peak as

$$
\hbar \omega \gtrsim 2 \Gamma,
$$

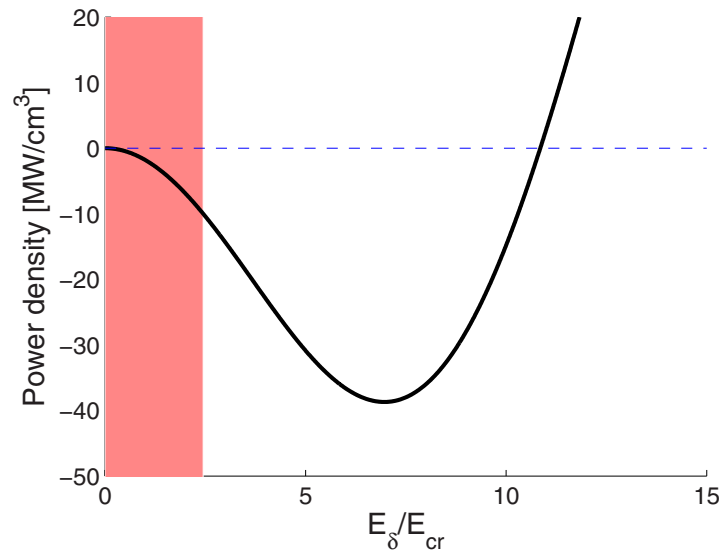

FIG. 3. (Color online) Generated power density as a function of the probe field amplitude $E_{\delta}$ for fixed $E_{d c}=5.5 E_{c r}$ and $\omega \tau=5$. The dark gray (red online) segment indicates the range of negative differential conductivity. If one assumes that the electric instability due to NDC at small probe amplitudes does not prevent the field in the resonator from growing, then the generation with a positive differential conductivity can be achieved for $E_{\delta} \in\left[2.5 E_{c}, 10.8 E_{c}\right]$. 


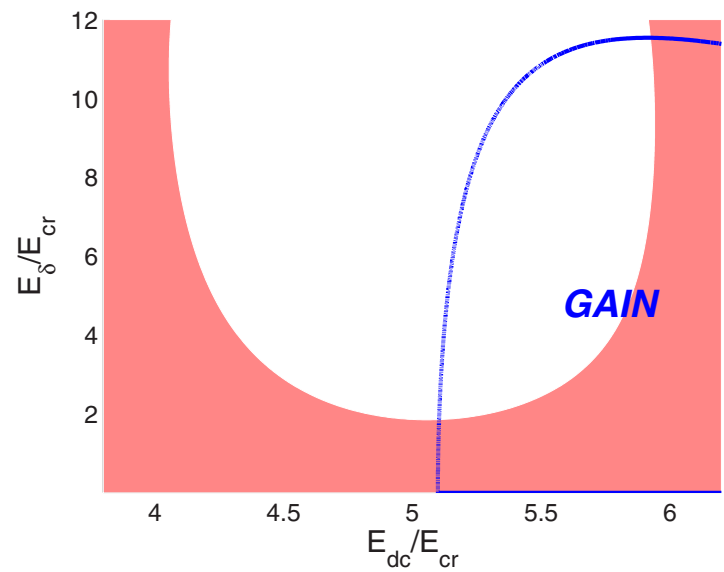

FIG. 4. (Color online) Regions of NDC (red online) and gain (marked area bounded by the blue curve) for $\omega \tau=5$. The region of gain overlaps with the region of positive differential conductivity (blank) for $5=\omega \tau<E_{d c} / E_{c r}<\omega \tau+1=6$ and $E_{\delta}>2 E_{c r}$. The figure indicates the possibility of reaching a large-signal amplification in a dc-biased SL without NDC.

$$
\begin{gathered}
E_{\delta} \gtrsim 2 E_{c r}, \\
\hbar \omega<\hbar \omega_{B}<\hbar \omega+\Gamma .
\end{gathered}
$$

Requirements (9a) and (9b) — which state that the photon energy must be larger than the scattering induced broadening and that the ac field strength must be no less than two critical fields-guarantee the formation of a sharply defined photonassisted peak. Note that in the limit opposite to Eq. (9a), the photon-assisted peaks never arise (see Ref. 41 and references cited therein). Finally, condition (9c) simultaneously ensures that (i) the absorption is negative, $\hbar \omega<\hbar \omega_{B}$, and (ii) the dc bias is chosen at the left wing of the photon-assisted peak corresponding to PDC $\left|\hbar \omega_{B}-\hbar \omega\right|<\Gamma$. For completeness, we should also mention that PDC and gain can be also simultaneously achieved by operating close to other photon-assisted peaks, $n \hbar \omega<\hbar \omega_{B}<n \hbar \omega+\Gamma(n=2,3, \ldots)$. In this case, gain and modifications in the dc current density occur due to multiphoton processes, and thus larger amplitudes of the probe field are required.

We should note that a dynamical response of electrons to terahertz radiation can depend on details of the scattering processes leading to the relaxation toward a thermal equilibrium. In the case of SLs with a single miniband, the simple model with two different scattering constants for the electron velocity $\gamma_{v}$ and electron energy $\gamma_{\varepsilon}$ often becomes more adequate than the approach based on a single relaxation time. ${ }^{42}$ Our numerical calculations indicate that inequalities (9a)-(9c) describe well the situation for $\gamma_{\varepsilon} / \gamma_{v} \geqslant 0.5$. However, a large-signal gain without NDC can still exist even if these scattering constants are very different (see the Appendix).

\section{SUPERLATTICE IN A BICHROMATIC FIELD}

We turn to the consideration of electron transport in a SL under the action of a bichromatic ac field

$$
E=E_{d c}+E_{a c} \cos \Omega t+E_{\delta} \cos \omega t .
$$

We suppose that the ac pump $E_{a c} \cos \Omega t$ is strong and the amplified probe field $E_{\delta} \cos \omega t$ in general can have an arbitrary amplitude. In the case of generation, the probe field is a mode of a resonator. We assume that the frequencies $\Omega$ and $\omega$ are incommensurate but both belong to the terahertz frequency domain $(\Omega \tau \gtrsim 1, \omega \tau \gtrsim 1)$.

In our earlier discussion, the probe field with a large amplitude induced some local structures into the VI characteristic of the SL, which eventually led to the large-signal gain without NDC. The main idea behind the bichromatic scheme of generation and amplification is to use an external ac field for the modification of the $V I$ characteristic, ${ }^{35,36}$ thus making it possible to amplify even a small probe signal without NDC. We will also show that fields with a large amplitude can be amplified in the same conditions as well.

\section{A. Main equations}

To begin with, we need to calculate the dc current density and absorption for the bichromatic field [Eq. (10)] with arbitrary amplitudes. By using the exact formal solution of the Boltzmann transport equation ${ }^{38}$ and following an earlier contribution to the formalism, ${ }^{12}$ we get $^{43,44}$ for the dc current

$$
j_{d c}^{\Omega \omega}=\sum_{k} J_{k}^{2}(\beta) j_{d c}^{\Omega}\left(e E_{d c} d+k \hbar \omega\right), \quad \beta=e E_{\delta} d / \hbar \omega,
$$

and the absorption of the probe field:

$$
\mathcal{A}=\frac{1}{2} \sum_{k} J_{k}(\beta)\left[J_{k+1}(\beta)+J_{k-1}(\beta)\right] j_{d c}^{\Omega}\left(e E_{d c} d+k \hbar \omega\right) .
$$

Here, the dc current density modified by the pump field alone is

$$
j_{d c}^{\Omega}\left(e E_{d c} d\right)=\sum_{l} J_{l}^{2}(\alpha) j_{d c}\left(e E_{d c} d+l \hbar \Omega\right)
$$

where $\alpha=e E_{a c} d / \hbar \Omega$. Next, the dc differential conductivity $\sigma_{d c}^{\Omega \omega}$ can be easily calculated from Eq. (11) as

$$
\sigma_{d c}^{\Omega \omega}=\sum_{l, k} J_{l}^{2}(\alpha) J_{k}^{2}(\beta) \sigma_{d c}\left(e E_{d c} d+l \hbar \Omega+k \hbar \omega\right) .
$$

NDC and PDC at the operation point $E_{d c}$ correspond to $\sigma_{d c}^{\Omega \omega}<0$ and $\sigma_{d c}^{\Omega \omega}>0$, respectively.

In the limit of a small probe field $(\beta \ll 1)$, from Eqs. (11) and (12), we have that $j_{d c}^{\Omega \omega} \approx j_{d c}^{\Omega}$ and

$$
\mathcal{A}=\frac{1}{2} \frac{j_{d c}^{\Omega}\left(e E_{d c} d+\hbar \omega\right)-j_{d c}^{\Omega}\left(e E_{d c} d-\hbar \omega\right)}{2 \hbar \omega} e E_{\delta} d,
$$

where $j_{d c}^{\Omega}$ is given by Eq. (13). Therefore, for a weak probe, both dc current and absorption are determined by the VI characteristic modified by the pump field. Since the formula for a small-signal gain in the bichromatic case [Eq. (15)] resembles the corresponding formula (5) after the substitution $j_{d c}^{\Omega} \rightarrow j_{d c}$, we can directly extend the simple geometric analysis of Sec. II A to the case of more complicated modi- 


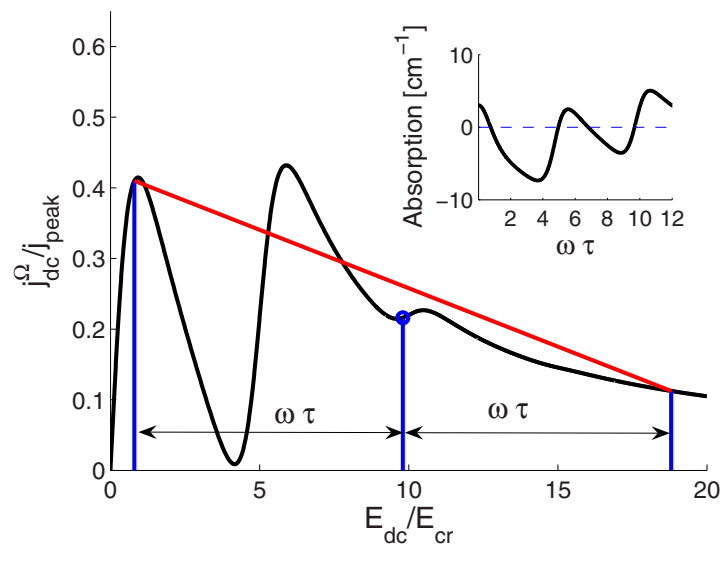

FIG. 5. (Color online) VI characteristic of SL under the action of a strong pump field $\left(\Omega \tau=5, E_{a c}=6 E_{c r}\right)$. In addition to the modified Esaki-Tsu peak, two photon-assisted peaks are visible. The operation point is chosen at the part of the second peak with a positive slope $\left(E_{d c}=9.8 E_{c r}\right)$. The quantum derivative [see Eq. (15)] at this operation point is negative for a wide range of $\omega$ values demonstrating the possibility of small-signal gain without NDC. Inset: Smallsignal absorption as a function of the probe frequency $\omega$ at $E_{d c}$ $=9.8 E_{c r}$. There are two distinct gain resonances at frequencies $\omega \tau$ $\approx 3.8$ and $\omega \tau \approx 8.8$.

fications of the VI characteristic caused by the action of a strong ac pump.

\section{B. Small-signal analysis: Basic ideas}

We want to show how to achieve a high-frequency gain working at parts of the modified VI characteristic with positive slopes. With this aim, we choose an ac pump with a large amplitude $\left(E_{a c} / E_{c r} \gg 1\right)$ and a high frequency $(\Omega \tau$ $\gg 1)$. For such pump fields, new local structures in the $V I$ characteristic become most pronounced and simple analytic formulas describing the modifications of the dc current and gain can be derived. Figure 5 illustrates the dependence of the dc current density on the dc bias, which has been calculated using Eq. (13) for $\hbar \Omega=5 \Gamma(\Omega \tau=5)$ and $E_{a c}=6 E_{c r}$. In addition to the modified Esaki-Tsu peak, one can see two new photon-assisted peaks. The peaks of the current are centered at

$$
E_{d c} / E_{c r} \approx n \Omega \tau+1,
$$

with $n=0,1,2$. The left wings of the photon-assisted peaks $\left(4<E_{d c} / E_{c r}<6\right.$ and $\left.9<E_{d c} / E_{c r}<11\right)$ have a positive slope, i.e., PDC. We found that PDC arises if the Wannier-Stark spacing approximately equals the energy of one photon, $\left|\hbar \omega_{B}-\hbar \Omega\right|<\Gamma\left(\Omega \tau-1<E_{d c} / E_{c r}<\Omega \tau+1\right)$, or the energy of two photons, $\left|\hbar \omega_{B}-2 \hbar \Omega\right|<\Gamma\left(2 \Omega \tau-1<E_{d c} / E_{c r}<2 \Omega \tau+1\right)$. In a more general case, the necessary condition for PDC becomes

$$
\left|\hbar \omega_{B}-n \hbar \Omega\right|<\Gamma \quad(n=0,1,2 \ldots) .
$$

The choice of the operation point at these parts of the $V I$ should prevent the development of space-charge instability typical for systems with NDC. For example, in Fig. 5, we choose the working point at the left part of the second photon-assisted peak.
Following Eq. (15), the calculation of the high-frequency absorption of a weak field requires the finding of the quantum derivative of $j_{d c}^{\Omega}$ at the working point $E_{d c}$. The geometric meaning of the quantum derivative, which is evident from Fig. 5, is similar to the one described in Sec. II A. Importantly, the quantum derivative can be negative in certain ranges of $\omega$ providing gain for these frequencies (Fig. 5).

It is easy to see that gain has a maximum if the left end of the segment is located at one of the dc current peaks. By taking into account that for high frequencies the segment has a small slope and the peaks are centered at $E_{d c}$ given by Eq. (16), we get that the gain resonances exist for the values of bias and probe frequency satisfying $E_{d c} / E_{c r}-\omega \tau \approx k \Omega \tau+1$ $(k=0,1,2)$. In terms of photon energies and the WannierStark spacing, this condition for the gain resonances takes the form

$$
\hbar \omega \approx \hbar \omega_{B}-k \hbar \Omega-\Gamma
$$

The inset of Fig. 5 shows the absorption as a function of $\omega$ for $\hbar \omega_{B}=9.8 \Gamma, \hbar \Omega=5 \Gamma$, and $E_{a c}=6 E_{c r}$. The gain profile demonstrates two distinct gain resonances at $\hbar \omega \approx 3.8 \Gamma$ and $\hbar \omega \approx 8.8 \Gamma$, which are described well by Eq. (18) with $k=1$ and $k=0$.

The dispersive gain profile and the locations of gain maxima indicate that from a pure quantum perspective, the gain in the dc-ac-driven SL originates from scatteringassisted transitions between quantum mechanical states. In contrast to the Bloch gain profile in a pure dc-biased SL, the photon sidebands also play a role here.

An interesting question is how the dispersive shape of the gain profile in the dc-ac-driven SL is sensitive to the amplitude and frequency of the pump field. Within the geometrical representation of the quantum derivative, a crossover from gain to absorption corresponds to the movement of the end of the virtual segment from peak to dip in the VI characteristic. Therefore, one can expect that the dispersive shape of the absorption profile near this crossover is universal as long as well pronounced local structures in VI do exist. Our numerical calculations indeed demonstrate that in the vicinity of the crossover point, which also includes the gain resonance, the gain profile remains almost unaltered.

Figure 6 shows the small-signal absorption as a function of $\omega$ for different amplitudes $\left(E_{a c} \in\left[0,3 E_{c r}\right]\right)$ of the highfrequency $(\hbar \Omega=5 \Gamma)$ pump field. We choose the operation point $E_{d c}=4.8 E_{c r}$. If the ac pump is strong enough, this operation point belongs to the first photon-assisted peak with PDC (see Fig. 5, where $E_{a c}=6 E_{c r}$ ). For all amplitudes, the dispersive Bloch gain profile at high frequencies is maintained together with the gain resonance at the photon energy $\hbar \omega \approx \hbar \omega_{B}-\Gamma=3.8 \Gamma$. However, with increasing pump amplitude, the low-frequency absorption changes from negative to positive; the switch from NDC to PDC takes place at $E_{a c}$ $\approx 2 E_{c r}$. Although the electric stability is achieved by a drastic change in the low-frequency absorption, there is practically no expense in the high-frequency gain.

To have an analytical formula describing the requirements for the existence of the Bloch gain at PDC in a dc-ac-driven SL, we directly combine the condition for PDC [Eq. (17)] 


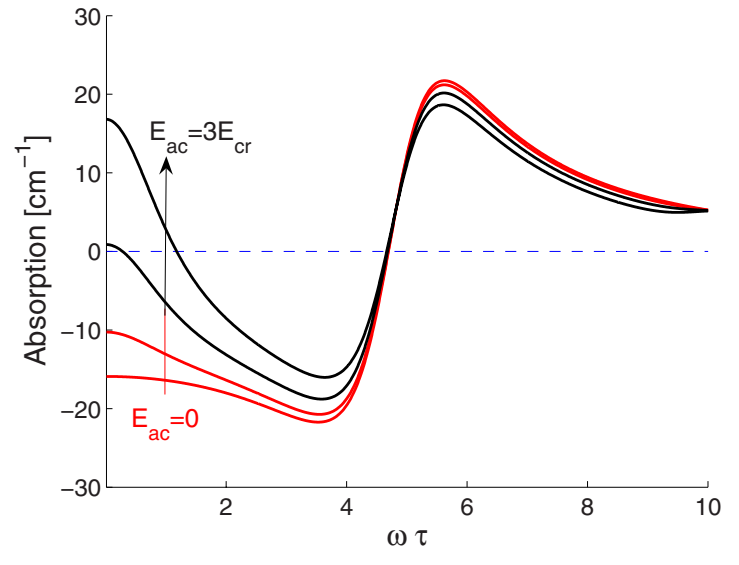

FIG. 6. (Color online) Small-signal absorption as a function of the probe frequency $\omega$ for $\Omega \tau=5$ and different values of the pump amplitude $E_{a c} / E_{c r}=: 0,1.2,2.1,3$. The gain profile near the maximum of gain only slightly changes with increase in the pump amplitude. However, the absorption at low frequencies changes from negative to positive indicating the transition from NDC (online red curves) to PDC (online black curves) at the operation point $E_{d c}$ $=4.8 E_{c r}$.

with the formula for gain resonances [Eq. (18)] and get the inequality $(n-k) \hbar \Omega-2 \Gamma<\hbar \omega=\hbar \omega_{B}-k \hbar \Omega-\Gamma<(n-k) \hbar \Omega$. It can be rewritten as

$$
|\hbar \omega-m \hbar \Omega+\Gamma|<\Gamma,
$$

where the index $m(m=1,2,3 \ldots)$ marks the $m$ th range of gain in the absorption profile. We numerically observed that this naive estimate surprisingly works well for highfrequency pump fields with amplitudes $E_{a c} \gtrsim 2 E_{c r}$. Here, the underlying physics is the robustness of the gain profile at high frequencies with its simultaneous sensitivity to variations in the pump field at low frequencies.

\section{General conditions for gain: Signals of arbitrary amplitude and positive dc conductivity}

We want to show how to extend our analysis to the case of a probe field which is not weak anymore. Our computations demonstrate that for parameters satisfying the smallsignal gain resonance, the large-signal gain as a rule also exists. Moreover, we found that the gain profile near gain resonances stays almost unaltered as the probe amplitude changes. Furthermore, the important condition (19) works also at large amplitudes of the probe field. However, simultaneously, the analog of Eq. (17) for the probe field must be also introduced: The Wannier-Stark spacing is approximately equal to an integer multiple of the photon energies at the probe frequency, $\left|\hbar \omega_{B}-l \hbar \omega\right|<\Gamma$.

Now, we can summarize our findings and list all necessary requirements to be satisfied in order to use the bichromatic scheme for the generation of a high-frequency field under conditions of electric stability:

$$
\begin{gathered}
\hbar \Omega \gtrsim 2 \Gamma, \\
E_{a c} \gtrsim 2 E_{c r},
\end{gathered}
$$

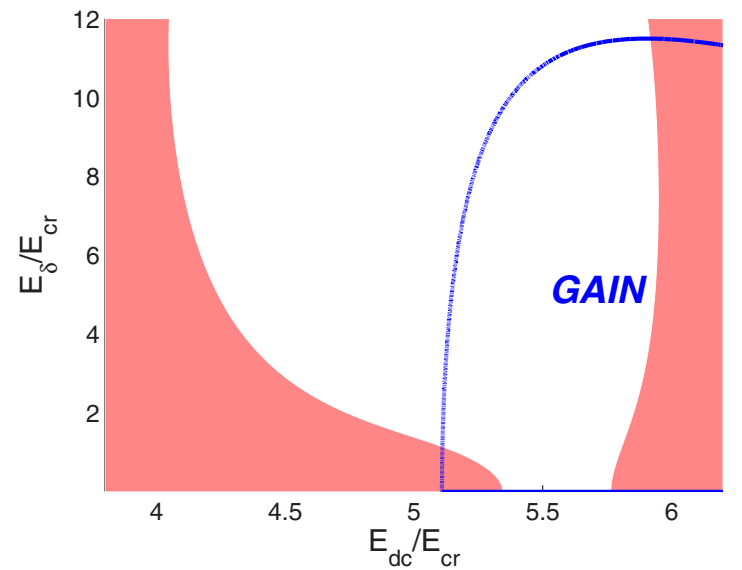

FIG. 7. (Color online) Regions of NDC (red online) and gain (marked area bounded by the blue curve) for $E_{a c}=2 E_{c r}, \Omega \tau=5.5$, and $\omega \tau=5$. The figure illustrates the feasibility of gain in conditions of suppressed electric instability for the wide ranges of signal amplitudes $E_{\delta}$ and bias values $E_{d c}$ under the action of a moderate ac pump.

$$
\begin{gathered}
n \hbar \Omega-\Gamma<\hbar \omega_{B}<n \hbar \Omega+\Gamma, \\
\hbar \omega \gtrsim 2 \Gamma, \\
l \hbar \omega-\Gamma<\hbar \omega_{B}<l \hbar \omega+\Gamma, \\
m \hbar \Omega-2 \Gamma<\hbar \omega<m \hbar \Omega,
\end{gathered}
$$

where, in general, integers $n, l$, and $m$ are different. Some of these inequalities, for example, Eqs. (20b) and (20c), are, of course, the same as derived earlier and are listed here for completeness. We should note that these conditions are asymptotic and work better the larger the amplitude [Eq. (20b)] and the higher the frequencies [Eqs. (20a) and (20d)].

Requirements (20a)-(20c) are necessary for the amplification of a weak probe field at an incommensurate frequency $\omega$ in conditions of PDC. They ensure that the operation point is located at the part of the well-defined $n$th photon-assisted peak with a positive slope. In order that the probe field can still be amplified even if its amplitude is not small anymore, requirements (20d)-(20f) should be taken into an account. Below, we will demonstrate how requirements (20a)-(20f) work in the cases of terahertz generation at the first and second photon-assisted peaks. The effect of different relaxation constants, $\gamma_{v} \neq \gamma_{\varepsilon}$, is analyzed in the Appendix.

\section{Gain and generated power density at the first photon-assisted peak}

Figure 7 shows the regions of gain and NDC for different values of dc bias and probe amplitude $E_{\delta}$ when the pump amplitude is fixed (cf. Fig. 4). It demonstrates the possibility of simultaneously achieving PDC and gain at the first photon-assisted peak $[n=1$ in Eq. (20c)] for the probe frequency in the proximity of the pump frequency $[m=1$ in Eq. (20f)]. In this figure, we choose a relatively low amplitude of the pump $E_{a c}=2 E_{c r}$, which is close to the threshold amplitude [see Eq. (20b)]. As a consequence, PDC for $E_{\delta} \rightarrow 0$ 

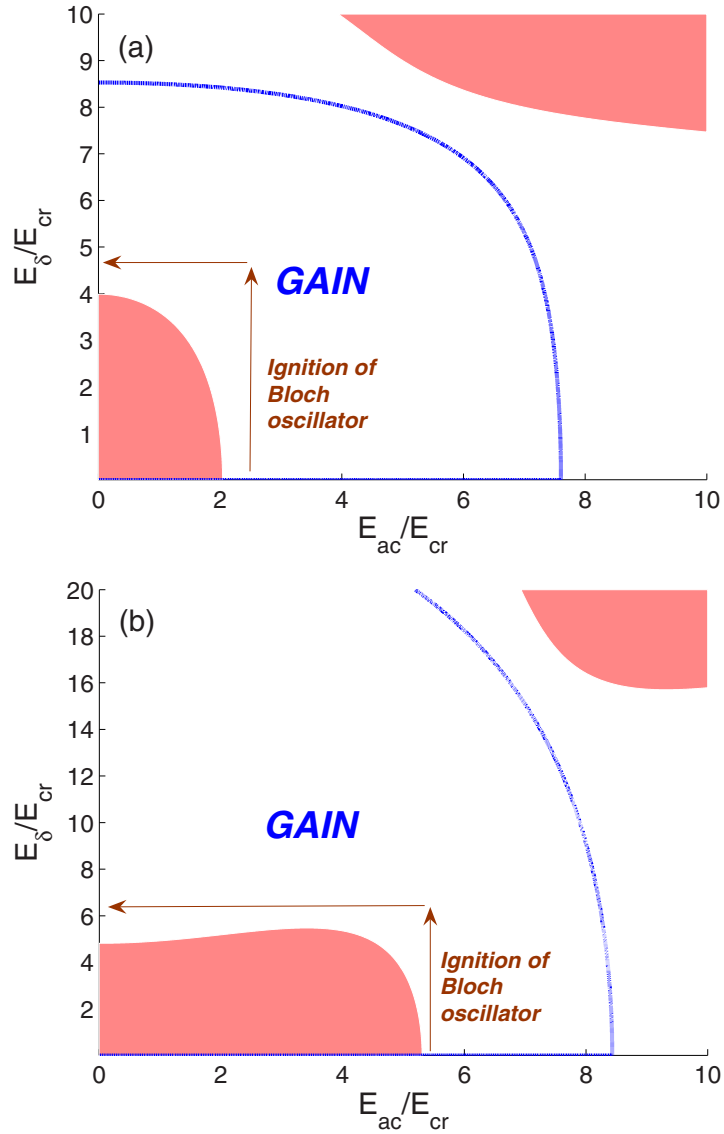

FIG. 8. (Color online) Regions of NDC (red online) and gain (marked) in the plane of pump-probe amplitudes for (a) $\Omega \tau=5$, $\omega_{B} \tau=4.8$ and $\omega \tau=4$ and (b) $\Omega \tau=5, \omega_{B} \tau=9.8$ and $\omega \tau=9$. Gain can exists without NDC. The arrows schematically illustrate the use of an ac pump in the terahertz starter for a Bloch oscillator (see Sec. IV for details).

exists for $5.3 E_{c r}<E_{d c}<5.8 E_{c r}$, which is a bit narrower than the range $4.5 E_{c r}<E_{d c}<6.5 E_{c r}$ following from Eq. (20c). On the other hand, Fig. 8(a) shows the regions of gain and NDC in the plane $E_{a c}-E_{\delta}$ for a fixed dc bias. It demonstrates that gain in conditions of PDC exists for the pump amplitudes $E_{a c} \in\left[2 E_{c r}, 7.6 E_{c r}\right]$, when requirements (20a)-(20f) are satisfied.

Figure 9 shows that the generated power density at the first photon-assisted peak in the bichromatic scheme slightly decreases with increase in the pump amplitude but still remains comparable to the power density that can be potentially generated in the canonic Bloch oscillator (cf. Fig. 3). Moreover, a comparison of Figs. 6 and 1 (inset) allows a similar statement concerning the magnitudes of gain in these two schemes to be made.

\section{Generation with frequency shifting}

By applying a very strong pump field, due to multiphoton processes, it is possible to exploit other photon-assisted peaks and reach the generation at frequencies significantly different from the pump frequency. In the case of the second photon-assisted peak $[n=2$ in Eq. (20c)], the frequency of the probe field can be easily shifted from the pump frequency so

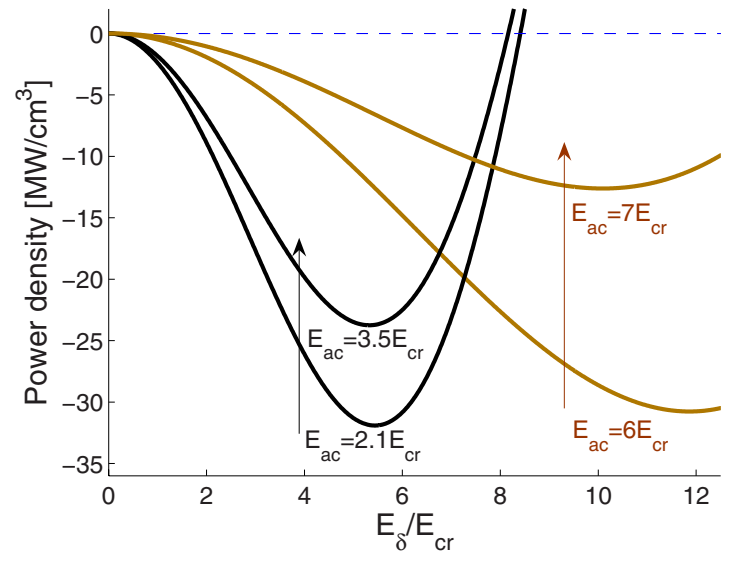

FIG. 9. (Color online) Generated power density as a function of the probe amplitude for several increasing values of the pump. The black curves correspond to the generation at the first photonassisted peak $\left(\Omega \tau=5, E_{d c}=4.8 E_{c r}, \omega \tau=4\right)$ induced by the ac pump with $E_{a c} / E_{c r}=: 2.1,3.5$. The gray (brown online) curves demonstrate that the generated power at the second photon-assisted peak $\left(\Omega \tau=5, E_{d c}=9.8 E_{c r}, \omega \tau=9\right)$ more strongly depends on the pump amplitude $\left(E_{a c} / E_{c r}=: 6,7\right)$. As can be seen from Fig. 8, the generation in both cases can be realized without the electric instability.

that the photon energy will be close to the gain resonance, $\hbar \omega \approx 2 \hbar \Omega-\Gamma$, corresponding to $m=2$ in Eq. (20f). On the other hand, requirement $(20 \mathrm{e})$ can be satisfied for $l=1$ by tuning the dc bias. Therefore, for a high enough frequency of the pump, all requirements, Eqs. (20a)-(20f) are satisfied, and amplification of the probe field with both small and large amplitudes can be achieved in conditions of PDC. Figures 5, 8(b), and 9 demonstrate this kind of possibilities for the pump frequency $\Omega \tau=5$ and dc bias $E_{d c}=9.8 E_{c r}$.

\section{TERAHERTZ STARTER FOR A BLOCH OSCILLATOR}

It is easy to notice that the large-signal gain in conditions of PDC is realized at the same parameters of probe fields in both the monochromatic and bichromatic schemes of amplification. A good illustration of this comes from the comparison of Figs. 4 and 7. They show the regions of gain and PDC in these two different schemes for the same photon energy of the probe field, $\hbar \omega=5 \Gamma$. In both cases, the regions of gain and PDC approximately overlap for dc bias values $5.1 E_{c r}$ $<E_{d c}<5.9 E_{c r}$ and probe amplitudes $1.9 E_{c r}<E_{\delta}<11.5 E_{c r}$. However, in the bichromatic case, there also exists an additional overlap region at small probe amplitudes for $5.3 E_{c r}$ $<E_{d c}<5.8 E_{c r}$ due to the formation of a photon-assisted peak induced by the action of the ac pump.

In a more general case, we can compare requirements (9a)-(9c) and (20a)-(20f) assuming that the probe frequency and dc bias satisfy conditions (9a) and (9c), we immediately see that conditions $(20 \mathrm{~d})$ and $(20 \mathrm{e})$ (with $l=1$ ) are also satisfied. Moreover, the rest of the requirements can be satisfied with a proper choice of the pump field.

Therefore, it is potentially possible to combine both these schemes in a single device. The process of a domain-free generation in a resonator with a very high $Q$ will include two stages: In the first stage, the ac pump is used to excite the 
field mode with an amplitude $E_{\delta} \gtrsim 2 E_{c r}$ [requirement (9a)]. In the second stage, the pump field is gradually switched off and sustained generation is performed at a large amplitude of $E_{\delta}$. We underline that here the whole switching off process takes place in conditions of a strong gain and PDC. Figure 8 illustrates these two stages of generation utilizing the first (a) and second (b) photon-assisted peaks. The arrows show a safe pass around the dangerous NDC region in the $E_{a c}-E_{\delta}$ plane, which corresponds to the ignition of the canonic Bloch oscillator.

Since the pump ac field is needed only in the initial stage in order to suppress the formation of electric domains and it is then switched off, it seems reasonable to use a terahertz pulse instead of a cw field in this terahertz starter for a Bloch oscillator. The required high-power pulses with $100 \mathrm{~ns}$ to $1 \mu$ s duration can be generated, for instance, with the help of a free-electron laser or a $\mathrm{CO}_{2}$ laser.

A quite different situation arises when few-cycle terahertz pulses are in use. The field cannot be considered as a monochromatic anymore. Therefore, we will now analyze the feasibility of gain in the case of a polychromatic ac pump. For simplicity, we consider the case of a trichromatic field

$$
E_{\text {pump }}^{a c}=\frac{E_{a c} \cos \Omega t}{2}+\frac{E_{a c} \cos \Omega_{1} t}{4}+\frac{E_{a c} \cos \Omega_{2} t}{4} .
$$

The amplified probe field continues to be monochromatic $E_{\delta} \cos \omega t$. We suppose that the pump, $\Omega, \Omega_{1}, \Omega_{2}$, and probe, $\omega$, frequencies satisfy the generalized incommensurability condition. Namely, we assume that there does not exist any nontrivial integers $m_{i}(i=1,2,3,4)$ such that $m_{1} \Omega_{1}+m_{2} \Omega_{2}$ $+m_{3} \Omega_{3}+m_{4} \omega=0$. Equation (21) can be considered as a crude approximation of the temporal shape of a real broadband terahertz pulse, where the Fourier transform of the pulse has a maximum at $\Omega$ and $\left|\Omega_{2}-\Omega_{1}\right|$ is related to the bandwidth of the pulse.

The analysis of previous sections can be directly generalized to the case of the polychromatic pump field [Eq. (21)], and we get for dc current density

$$
j_{d c}^{\Omega \Omega_{1} \Omega_{2} \omega}=\sum_{k} j_{k}^{2}(\beta) j_{d c}^{\Omega \Omega_{1} \Omega_{2}}\left(e E_{d c} d+k \hbar \omega\right)
$$

and for absorption of the probe field, we get

$$
\mathcal{A}=\frac{1}{2} \sum_{k} J_{k}(\beta)\left[J_{k+1}(\beta)+J_{k-1}(\beta)\right] j_{d c}^{\Omega \Omega_{1} \Omega_{2}}\left(e E_{d c} d+k \hbar \omega\right) .
$$

Here, $j_{d c}^{\Omega \Omega_{1} \Omega_{2}}$ is the dc current density in the SL modified by the pump field alone:

$$
\begin{aligned}
j_{d c}^{\Omega \Omega_{1} \Omega_{2}}= & \sum_{l, m, n} J_{l}^{2}(\alpha / 2) J_{m}^{2}(\alpha / 4) J_{n}^{2}(\alpha / 4) \\
& \times j_{d c}\left(e E_{d c} d+l \hbar \Omega+m \hbar \Omega_{1}+n \hbar \Omega_{2}\right) .
\end{aligned}
$$

Equations (22) and (23) are valid for an arbitrary amplitude of the probe field. Remarkably, in the limit of a weak probe field $\left(E_{\delta} \rightarrow 0\right)$, the absorption $\mathcal{A}$ becomes proportional to the quantum derivative of the pump-modified dc current [Eq. (24)].

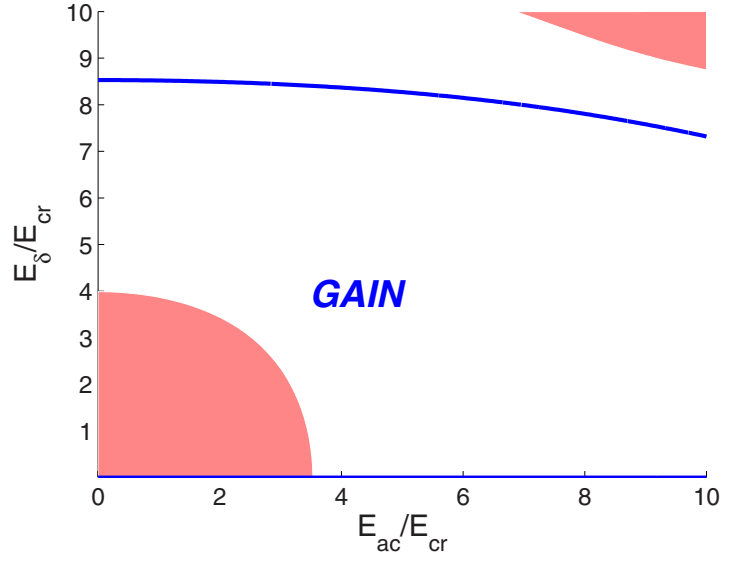

FIG. 10. (Color online) Regions of NDC (red online) and gain (marked) for $\omega_{B} \tau=4.8, \omega \tau=4$, and the trichromatic pump field [Eq. (21)] with $\Omega \tau=5, \Omega_{1} \tau=5.5$, and $\Omega_{2} \tau=4.75$. The comparison of this figure with Fig. 8(a) demonstrates that the realization of gain in conditions of PDC under the polychromatic pump requires larger values of $E_{a c}$ than in the case of a monochromatic pump.

In the limit $\Omega_{i} \rightarrow \Omega(i=1,2)$, the trichromatic pump field [Eq. (21)] becomes monochromatic, and therefore, one would expect restoration of all results of Sec. III. However, that is not the case, and the current density and absorption, which are given by Eqs. (22) and (23) are not reduced to the corresponding equations of Sec. III. The disagreement arises because the condition of incommensurability is not satisfied in this limit.

We computed the regions of NDC and gain for the pump [Eq. (21)] with two different side frequencies $\Omega_{1}$ and $\Omega_{2}$ when the cental frequency $\Omega$ determines the first photonassisted peak in the $V I$ characteristic. Figure 10 shows the regions of gain and PDC in the $E_{a c}-E_{\delta}$ plane. The WannierStark spacing and photon energies $\left(\hbar \omega_{B}=4.8 \Gamma, \hbar \Omega=5 \Gamma\right.$, and $\hbar \omega=4 \Gamma)$ are the same as used in Fig. 8(a). By comparing Figs. 8 and 10, one observes that the gain and PDC can be achieved in both cases at similar amplitudes of the probe field, while a bit larger pump is required in the trichromatic case.

These results support the possibility of replacing a monochromatic pump with a broadband terahertz pulse in the starter of a Bloch oscillator. Required intensive $\mathrm{T}$ rays can be produced by a number of ways, including the use of an interdigitated photoconducting device, ${ }^{45}$ amplifier systems ${ }^{46}$ or an ultrafast ionizing air $^{47}$ (for a recent review, see Ref. 37).

\section{CONCLUSION}

We theoretically analyzed the feasibility of reaching terahertz gain in a dc-biased semiconductor superlattice at room temperature by using mono- and polychromatic alternating pump fields. We showed that different kinds of pump fields can suppress the formation of high-field electric domains inside the superlattice while still preserving a broadband terahertz gain at frequencies incommensurate with the pump frequencies. 
Our approach is based on the utilization of well-known photon-assisted peaks $\mathrm{s}^{35,36}$ arising in the voltage-current characteristics of terahertz-driven superlattices. The choice of the operation point at the positive slope of such a peak allows the suppression of the electric instability. For the search of a high-frequency gain in conditions of the positive differential conductivity, we employed a simple but powerful geometric interpretation of the intraminiband absorption formulas. By combining these analytic tools and numerical simulations, we demonstrated that the Bloch gain profile in a dc-ac-driven superlattice is robust near the gain resonances.

We suggested the use of the robustness property of terahertz gain in an effective starter for the canonic (only dcbiased) Bloch oscillator operating in the electrically stable large-amplitude mode of generation. ${ }^{20}$ Since the terahertz gain in both the canonic Bloch oscillator and dc-ac-pumped Bloch oscillator occurs in the same ranges of dc bias, generation frequency, and amplitude, a temporal application of the ac pump field can be used for a domain-free generation up to the amplitude, which becomes sufficient for a stable operation of the canonic Bloch oscillator. The pump field, which ignites the Bloch oscillator, can also be polychromatic. We predicted that available intensive, broadband terahertz pulses ( $\mathrm{T}$ rays) can be potentially used in the fast ignition of the Bloch oscillator.

We conclude with a few remarks. First, in this work, we limit our attention to the case of incommensurate frequencies. If the pump and probe frequencies are commensurate, an additional coherent term in the formulas for a highfrequency absorption will arise. ${ }^{48}$ The sign of the term depends on the relative phase between the pump and amplified fields. ${ }^{48,49}$ However, at least in the case of generation with the monochromatic pump field, the coherent term always contributes to the high-frequency gain. A more detailed analysis of this situation, including consideration of microwave and subterahertz pump fields, ${ }^{50,51}$ will be presented elsewhere.

Second, we have focused on the local modification of the voltage-current characteristics of superlattices arising when the Bloch frequency approaches the ac field frequency or its harmonics. However, similar modifications of the electric characteristics have been observed in the semiconductor superlattices subject to a tilted high magnetic field when the Bloch frequency is close to the cyclotron frequency or its harmonics ${ }^{52}$ It is an interesting problem to consider modifications of the voltage-current characteristics ${ }^{53}$ and analyze the feasibility of terahertz gain in a superlattice under the combined action of the tilted magnetic and alternating electric fields.

Finally, we note that our formulas for dc current in the form of photon replicas of the VI characteristic of strongly coupled superlattice driven by the monochromatic [Eq. (6)], bichromatic [Eq. (11)], or polychromatic [Eq. (22)] ac fields are identical to the corresponding formulas for photonassisted dc current of Tien and Gordon ${ }^{55}$ and its direct generalization to the case of a multimode drive. ${ }^{56}$ Therefore, we speculate that our method for suppression of NDC-related instabilities by application of additional high-frequency ac fields can be directly extended to other tunneling structures, including various semiconductor devices.

\section{ACKNOWLEDGMENTS}

We thank Ahti Leppänen for cooperation, Andreas Wacker for bringing Ref. 33 to our attention and Amalia Patane, Alexander Balanov, Manfred Helm, Anatoly Ignatov, Miron Kagan, Alvydas Lisauskas, Jussi Mattas, Hartmut Roskos, Harald Schneider, Alexey Shorokhov, Gintaras Valušis, and Stephan Winnerl for useful discussions. We are grateful to Feodor Kusmartsev for numerous advices and constant encouragement of this activity within the EU program. This research was partially supported by the Emil Aaltonen Foundation, Academy of Finland (Nos. 101165 and 109758), Magnus Ehrnrooth Foundation, Väisälä Foundation, and AQDJJ Programme of the European Science Foundation.

\section{APPENDIX: EFFECT OF ELASTIC SCATTERING}

Here, we consider the effects of different scattering constants in both the monochromatic [Eq. (2)] and bichromatic [Eq. (10)] schemes of terahertz amplification and generation. The following SL balance equations can be derived from the Boltzmann transport equation for a single miniband of the SL: 6,42

$$
\begin{gathered}
\frac{d}{d t} j(t)+\frac{d^{2} e^{2} E(t)}{\hbar^{2}} \varepsilon(t)=-\gamma_{v} j(t), \\
\frac{d}{d t} \varepsilon(t)-E(t) j(t)=-\gamma_{\varepsilon}\left[\varepsilon(t)-\varepsilon_{\mathrm{eq}}\right],
\end{gathered}
$$

where $j(t)$ is the current density and $\varepsilon(t)$ is the total energy density of electrons within the miniband, $\gamma_{\varepsilon}$ and $\gamma_{v}=\gamma_{\varepsilon}$ $+\gamma_{e l}$ are the phenomenological scattering constants for electron energy and miniband electron velocity, and $\gamma_{e l}$ is the scattering constant describing elastic scattering events. All electrons are at the bottom of the miniband for $\varepsilon=-N \Delta / 2$ and the upper edge of the miniband is reached if $\varepsilon=$ $+N \Delta / 2$. The average electron energy in thermal equilibrium $\varepsilon_{\text {eq }}$ depends on the lattice temperature. ${ }^{6}$ It is convenient to introduce the mean scattering time $\tau=1 / \sqrt{\gamma_{v} \gamma_{\varepsilon}}$ and the ratio of scattering constants $\nu=\gamma_{\varepsilon} / \gamma_{v} \leqslant 1$. According to many experiments, ${ }^{54} \nu \gtrsim 0.5$ is a good assumption, although it is not valid for all superlattices. ${ }^{26}$

By numerically solving the SL balance equations [Eq. (A1)], we determine the time dependence of a steady current for $t \gg \tau$ and then calculate the dc differential conductivity and absorption of the probe field. In the case $\nu=1$, we find excellent agreement with the earlier analytical results following from formulas (7), (8), (12), and (14). We observe that the photon-assisted peaks occur in the VI characteristics for all values of $\nu \geqslant 0.1$; however, with a decrease in the ratio $\nu$, these VI structures become less pronounced and the magnitudes of the high-frequency gain are decreasing.

Importantly, the amplification still occurs in approximately the same ranges of field amplitudes for different values of $\nu$. Figure 11 represents the ranges of dc bias $E_{d c}$ and probe amplitude, supporting a large-signal gain with PDC at the first photon-assisted peak induced by the monochromatic field [Eq. (2)]. With decreasing $\nu$, the range of amplitudes for negative absorption shrinks but the range of amplitudes sup- 

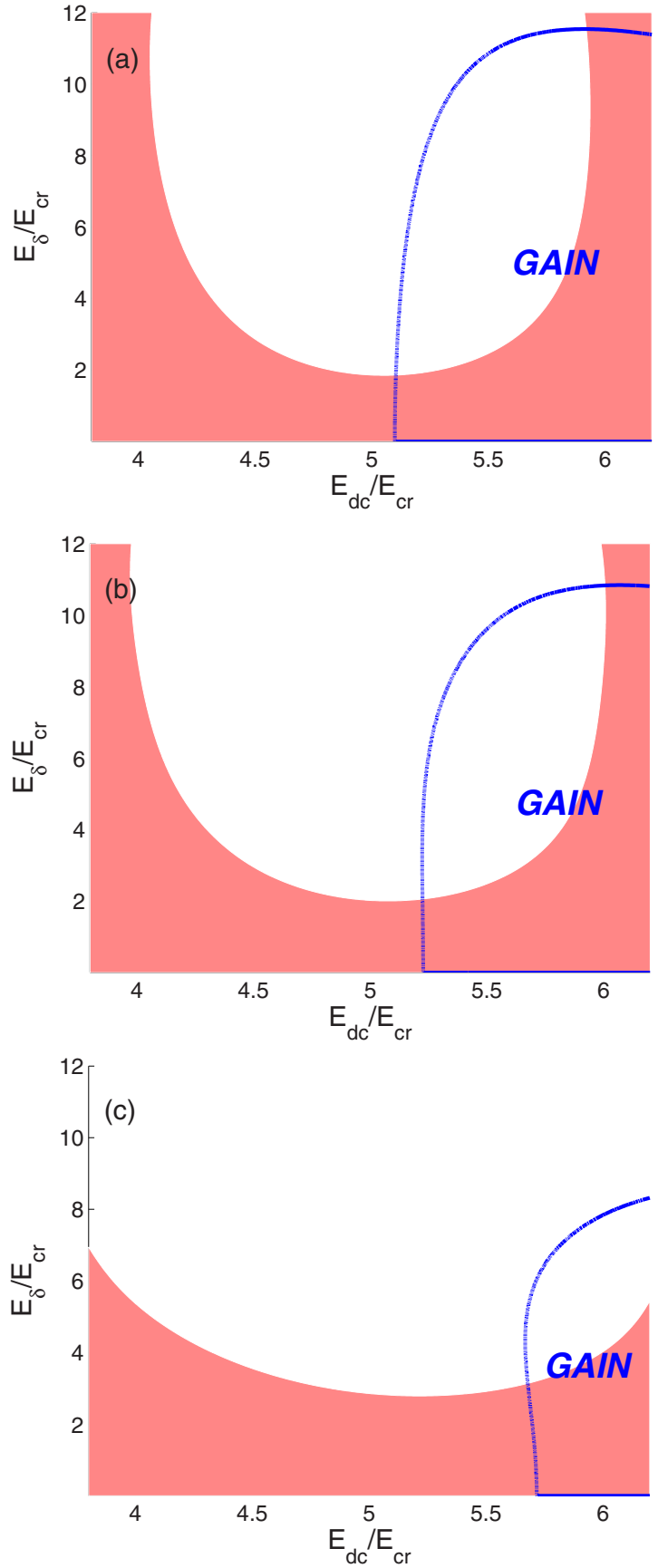

FIG. 11. (Color online) Regions of NDC (red online) and gain (marked) in dc-biased superlattice [Eq. (2)] for the probe field with the frequency $\omega \tau=5$ and for several values of $\nu$ : (a) $\nu=1$, (b) $\nu$ $=0.4$, and (c) $\nu=0.1$. The plots illustrate the feasibility of a largesignal amplification under suppressed electric instability for various ratios of the scattering constants.

porting PDC expands (cf. subplots in Fig. 11). The changes are obviously small as long as $\nu \geqslant 0.4$. Figure 12 shows the ranges of pump $E_{a c}$ and probe $E_{\delta}$ field amplitudes supporting a generation at the first photon-assisted peak in the bichro-
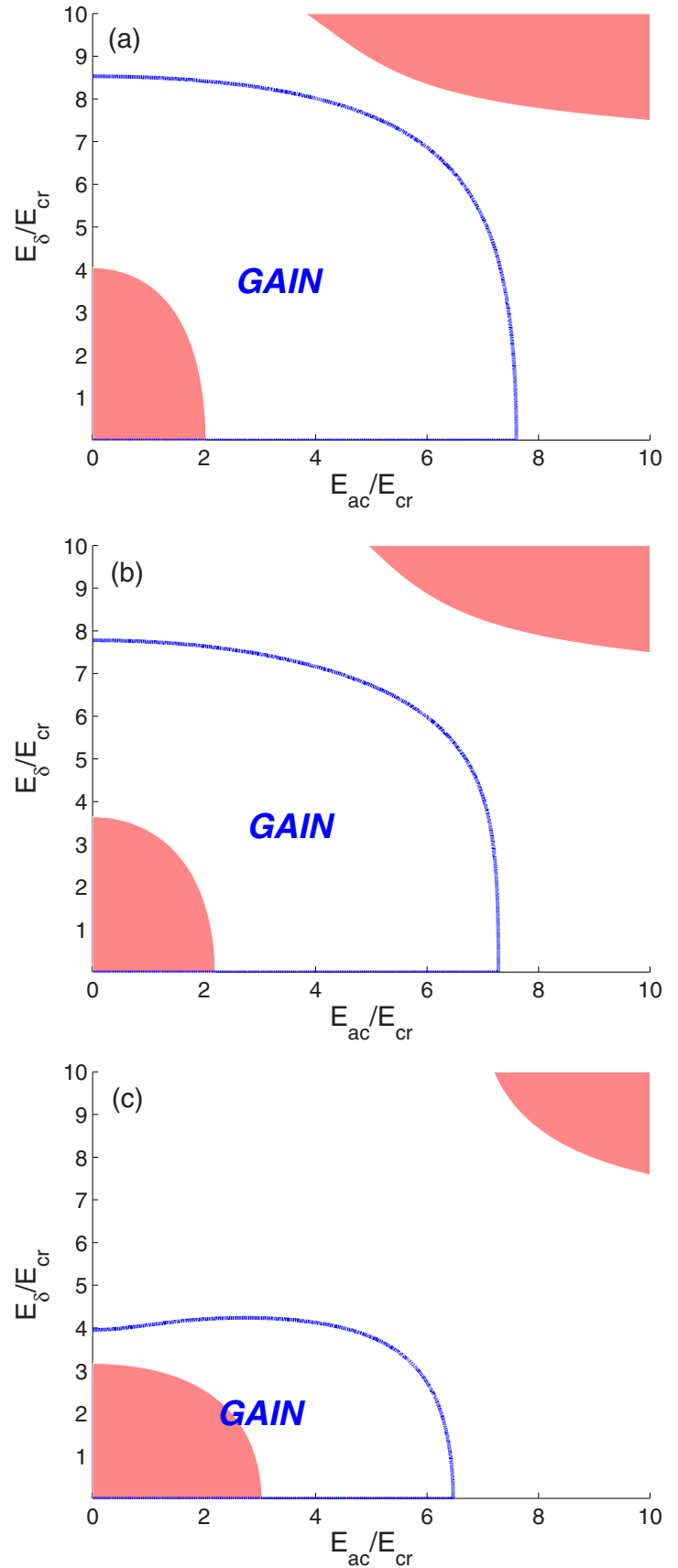

FIG. 12. (Color online) Regions of NDC (red online) and gain (marked) in an ac-pumped superlattice [Eq. (10)] for $\Omega \tau=5, \omega \tau$ $=4, \omega_{B} \tau=4.8$, and several values of $\nu$ : (a) $\nu=1$, (b) $\nu=0.4$, and (c) $\nu=0.1$. The plots illustrate the feasibility of terahertz generation under suppressed electric instability for various ratios of the scattering constants.

matic scheme. The suppression of electric domains and the absorption in the bichromatic field [Eq. (10)] are based on the same physical phenomena. Therefore, the decrease in $\nu$ affects the bichromatic scheme in the way as in the mochromatic case. 
${ }^{1}$ Proc. IEEE 80 (11) (1992); P. H. Siegel, IEEE Trans. Microwave Theory Tech. 50, 910 (2002); B. Ferguson and X.-C. Zhang, Nat. Mater. 1, 26 (2002); Philos. Trans. R. Soc. London, Ser. A 362(1815) (2004); M. Tonouchi, Nat. Photonics 1, 97 (2007).

${ }^{2}$ R. Köhler, A. Tredicucci, F. Beltram, H. E. Beere, E. H. Linfield, A. G. Davies, D. A. Ritchie, R. C. Iotti, and F. Rossi, Nature (London) 417, 156 (2002).

${ }^{3}$ S. Kumar, B. S. Williams, S. Kohen, Q. Hu, and J. L. Reno, Appl. Phys. Lett. 84, 2494 (2004); B. S. Williams, S. Kumar, Q. $\mathrm{Hu}$, and J. Reno, Opt. Express 13, 3331 (2005).

${ }^{4}$ L. Esaki and R. Tsu, IBM J. Res. Dev. 14, 61 (1970).

${ }^{5}$ F. G. Bass and A. P. Tetervov, Phys. Rep. 140, 237 (1986).

${ }^{6}$ A. Wacker, Phys. Rep. 357, 1 (2002).

${ }^{7}$ G. Platero and R. Aguado, Phys. Rep. 395, 1 (2004).

${ }^{8}$ F. Bloch, Z. Phys. 52, 555 (1928).

${ }^{9}$ C. Zener, Proc. R. Soc. London, Ser. A 145, 523 (1934).

${ }^{10}$ K. Leo, P. H. Bolivar, F. Bruggemann, R. Schwedler, and K. Köhler, Solid State Commun. 84, 943 (1992); J. Feldmann, K. Leo, J. Shah, D. A. B. Miller, J. E. Cunningham, T. Meier, G. von Plessen, A. Schulze, P. Thomas, and S. Schmitt-Rink, Phys. Rev. B 46, 7252 (1992); T. Dekorsy, P. Leisching, K. Köhler, and H. Kurz, ibid. 50, 8106 (1994).

${ }^{11}$ S. A. Ktitorov, G. S. Simin, and V. Ya. Sindalovskii, Fiz. Tverd. Tela (Leningrad) 13, 2230 (1971). [Sov. Phys. Solid State 13, 1872 (1972)].

${ }^{12}$ Yu. A. Romanov, V. P. Bovin, and L. K. Orlov, Fiz. Tekh. Poluprovodn. (S.-Peterburg) 12, 1665 (1978). [Sov. Phys. Semicond. 12, 987 (1978)].

${ }^{13}$ A. A. Ignatov, K. F. Renk, and E. P. Dodin, Phys. Rev. Lett. 70, 1996 (1993).

${ }^{14}$ H. Kroemer, arXiv:cond-mat/0007482 (unpublished); E. Schomburg, N. V. Demarina, and K. F. Renk, Phys. Rev. B 67, 155302 (2003).

${ }^{15}$ H. Willenberg, G. H. Döhler, and J. Faist, Phys. Rev. B 67, 085315 (2003); A. Wacker, ibid. 66, 085326 (2002).

${ }^{16}$ R. Terazzi, T. Gresch, M. Giovannini, N. Hoyler, N. Sekine, and J. Faist, Nat. Phys. 3, 329 (2007).

${ }^{17}$ J. B. Gunn, Solid State Commun. 1, 88 (1963); J. B. Gunn, IBM J. Res. Dev. 8, 141 (1964).

${ }^{18}$ M. Büttiker and H. Thomas, Phys. Rev. Lett. 38, 78 (1977); A. A. Ignatov and V. I. Shashkin, Zh. Eksp. Teor. Fiz. 93, 935 (1987) [Sov. Phys. JETP 66, 526 (1987)].

${ }^{19}$ H. Le Person, C. Minot, L. Boni, J. F. Palmier, and F. Mollot, Appl. Phys. Lett. 60, 2397 (1992); K. Hofbeck, J. Grenzer, E. Schomburg, A. A. Ignatov, K. F. Renk, D. G. Pavel'ev, Yu. Koschurinov, B. Melzer, S. Ivanov, S. Schaposchnikov, and P. S. Kop'ev, Phys. Lett. A 218, 349 (1996).

${ }^{20}$ H. Kroemer, arXiv:cond-mat/0009311 (unpublished).

${ }^{21}$ J. Copeland, J. Appl. Phys. 38, 3096 (1967).

${ }^{22}$ I. V. Altukhov, M. S. Kagan, S. G. Kalashnikov, V. V. Kukushkin, and V. N. Solyakov, Fiz. Tekh. Poluprovodn. (S.-Peterburg) 13, 2316 (1979) [Sov. Phys. Semicond. 13, 1356 (1979)].

${ }^{23}$ V. N. Sokolov, K. W. Kim, V. A. Kochelap, and D. L. Woolard, J. Appl. Phys. 98, 064507 (2005).

${ }^{24}$ P. G. Savvidis, B. Kolasa, G. Lee, and S. J. Allen, Phys. Rev. Lett. 92, 196802 (2004).

${ }^{25}$ A. Lisauskas, C. Blöser, R. Sachs, H. G. Roskos, A. Juozapavičius, G. Valušis, and K. Köhler, Appl. Phys. Lett. 86, 102103 (2005)

${ }^{26}$ N. Sekine and K. Hirakawa, Phys. Rev. Lett. 94, 057408 (2005).
${ }^{27}$ T. Kurosawa and S. Nagahashi, J. Phys. Soc. Jpn. 45, 707 (1978); A. A. Ignatov, Dokl. Akad. Nauk SSSR 273, 1351 (1983). [Sov. Phys. Dokl. 28, 1046 (1983)].

${ }^{28}$ T. Feil, R. A. Deutschmann, W. Wegscheider, M. Rother, D. Schuh, M. Bichler, G. Abstreiter, B. Rieder, and J. Keller, Phys. Status Solidi C 1, 2111 (2004).

${ }^{29}$ E. S. Daniel, B. K. Gilbert, J. S. Scott, and S. J. Allen, IEEE Trans. Electron Devices 50, 2434 (2003).

${ }^{30}$ T. Feil, H.-P. Tranitz, M. Reinwald, and W. Wegscheider, Appl. Phys. Lett. 87, 212112 (2005); T. Feil, C. Gerl, and W. Wegscheider, Phys. Rev. B 73, 125301 (2006).

${ }^{31}$ Yu. A. Romanov, L. G. Mourokh, and N. J. M. Horing, J. Appl. Phys. 93, 4696 (2003); G. M. Shmelev, I. I. Maglevanny, and E. M. Epshtein, J. Phys. A: Math. Theor. 41, 075002 (2008); M. L. Orlov, Yu. A. Romanov, and L. K. Orlov, Microelectron. J. 36, 396 (2005).

${ }^{32}$ D. A. Ryndyk, N. V. Demarina, J. Keller, and E. Schomburg, Phys. Rev. B 67, 033305 (2003).

${ }^{33}$ A. Wacker, S. J. Allen, J. S. Scott, M. C. Wanke, and A.-P. Jauho, Phys. Status Solidi B 204, 95 (1997).

${ }^{34}$ J. R. Tucker, IEEE J. Quantum Electron. 15, 1234 (1979).

${ }^{35}$ For the theory, see V. V. Pavlovich and É. M. Épshtein, Fiz. Tekh. Poluprovodn. (S.-Peterburg) 10, 2001 (1976) [Sov. Phys. Semicond. 10, 1196 (1976)]; F. G. Bass and E. A. Rubinshtein, Fiz. Tverd. Tela (Leningrad) 19, 1379 (1977) [Sov. Phys. Solid State 19, 800 (1977)].

${ }^{36}$ For the experiment, see K. Unterrainer, B. J. Keay, M. C. Wanke, S. J. Allen, D. Leonard, G. Medeiros-Ribeiro, U. Bhattacharya, and M. J. W. Rodwell, Phys. Rev. Lett. 76, 2973 (1996).

${ }^{37}$ K. Reimann, Rep. Prog. Phys. 70, 1597 (2007).

${ }^{38}$ R. G. Chambers, Proc. Phys. Soc., London, Sect. A 65, 458 (1952); H. Budd, Phys. Rev. 127, 4 (1962); C. MacCallum, ibid. 132, 930 (1963).

${ }^{39}$ G. Brozak, M. Helm, F. DeRosa, C. H. Perry, M. Koza, R. Bhat, and S. J. Allen, Phys. Rev. Lett. 64, 3163 (1990).

${ }^{40}$ A. A. Ignatov, E. Schomburg, J. Grenzer, S. Winnerl, K. F. Renk, and E. P. Dodin, Superlattices Microstruct. 22, 15 (1997).

${ }^{41}$ F. Klappenberger, K. N. Alekseev, K. F. Renk, R. Scheuerer, E. Schomburg, S. J. Allen, G. R. Ramian, J. S. S. Scott, A. Kovsh, V. Ustinov and A. Zhukov, Eur. Phys. J. B 39, 483 (2004).

${ }^{42}$ A. A. Ignatov and Yu. A. Romanov, Phys. Status Solidi B 73, 327 (1976); A. A. Ignatov, E. P. Dodin, and V. I. Shashkin, Mod. Phys. Lett. B 5, 1087 (1991).

${ }^{43}$ A. V. Shorokhov and K. N. Alekseev, Physica E (Amsterdam) 33, 284 (2006).

${ }^{44}$ T. Hyart, M.Sc. Thesis, University of Oulu, 2005.

${ }^{45}$ A. Dreyhaupt, S. Winnerl, M. Helm, and T. Dekorsy, Opt. Lett. 31, 1546 (2006).

${ }^{46}$ T. Bartel, P. Gaal, K. Reimann, M. Woerner, and T. Elsaesser, Opt. Lett. 30, 2805 (2005);

${ }^{47}$ K.-Y. Kim, J. H. Glownia, A. J. Taylor, and G. Rodriguez, Opt. Express 15, 4577 (2007).

${ }^{48}$ T. Hyart, A. V. Shorokhov, and K. N. Alekseev, Phys. Rev. Lett. 98, 220404 (2007).

${ }^{49}$ T. Hyart, N. V. Alexeeva, A. Leppänen, and K. N. Alekseev, Appl. Phys. Lett. 89, 132105 (2006).

${ }^{50}$ K. N. Alekseev, M. V. Gorkunov, N. V. Demarina, T. Hyart, N. V. Alexeeva, and A. V. Shorokhov, Europhys. Lett. 73, 934 (2006); 74, 567(E) (2006). 
${ }^{51}$ Yu. A. Romanov, J. Yu. Romanova, and L. G. Mourokh, J. Appl. Phys. 99, 013707 (2006).

${ }^{52}$ T. M. Fromhold, A. Patane, S. Bujkievicz, P. B. Wilkinson, D. Powler, D. Sherwood, S. P. Stapleton, A. A. Krokhin, L. Eaves, M. Henini, N. S. Sankeshwar, and F. W. Sheard, Nature (London) 428, 726 (2004).

${ }^{53}$ F. G. Bass, V. V. Zorchenko, and V. V. Shashora, Pis'ma Zh. Eksp. Teor. Fiz. 31, 345 (1980) [JETP Lett. 31, 314 (1980)].

${ }^{54}$ E. Schomburg, T. Blomeier, K. Hofbeck, J. Grenzer, S. Brandl, I.
Lingott, A. A. Ignatov, K. F. Renk, D. G. Pavel'ev, Yu. Koschurinov, B. Ya. Melzer, V. M. Ustinov, S. V. Ivanov, A. Zhukov, and P. S. Kop'ev, Phys. Rev. B 58, 4035 (1998).

${ }^{55}$ P. K. Tien and J. P. Gordon, Phys. Rev. 129, 647 (1963).

${ }^{56}$ For the case of a monochromatic ac field, the equivalence of $\mathrm{dc}$ current formulas obtained from the solution of the Boltzmann equation and within the Tien-Gordon approach is well known (Ref. 6). For the case of a polychromatic field, it has been recently proven: K. N. Alekseev and T. Hyart (unpublished). 